COMMENT. PET studies of human brain functional development have shown that the prefrontal and temporoparietal regions are relatively inactive before the sixth month of $1 \mathrm{ife}$ and an acult pattern is seen at one year of age. (Chugani HT et al. Ann Neurol 1987; 22:487-497). The theory of developmental arrest in infants with Rett syndrome is supported by the finding of an immature pattern of cerebral blood flow. However, the results of the present study conflict with those of Naidu et al who found an increase in the metabolism in the frontal region and a lower metabolism in the occipital region of two patients with Ret syndrome studied with PET (J Child Neurol 1988; $\underline{3}$ (suppl): S78-S86).

SURAL NERVE AXONOPATHY AND RETT SYNDROME

The histopathologic findings of three sural nerve biopsies and one muscle biopsy from three patients with Rett syndrome are described from the Department of Pediatrics, National Sanatorium Yakumo Hospital, Yakumo, Hokkaido, Japan. The biopsies demonstrated mitochondrial changes in the cytoplasm of Schwann cells, occasional onion bulb formations, and mitochondrial alterations in myelinated axons with reduction in the number of large myelinated fibers. The muscle showed small dark angulated fibers with $\mathrm{NADH}-\mathrm{TR}$ staining and dumbbell-shaped mitochondria. (Wakai $\mathrm{S}$ et al. Rett syndrome: Findings suggesting axonopathy and mitochondrial abnormalities. Pediatr Neurol Sept/Oct $1990 ; \underline{6}: 339-343)$.

COMNENT. These findings suggest peripheral nerve involvement and mitochondrial abnormalities in Rett syndrome. An additional article describes cerebellar pathology at autopsy of five patients with Rett syndrome (Oldfors A et al. Ret syndrome: Cerebellar pathology. Pediatr Neurol Sept/Oct 1990; 6:310-314). Patients ranged in age fram $7 \overline{\text { to } 30}$ years. All had reduced brain weights with small cerebella. There was loss of Purkinje cells, atrophy, astrocytic gliosis of molecular and granular cell layers, and gliosis and loss of myelin in the white matter. The cerebellar atrophy was greater in two patients treated with phenytoin. The pathology of Rett syndrome appears to involve the cerebellum, cerebral hemispheres, basal ganglia, especially substantia nigra, spinal cord, peripheral nerve, and muscle.

\title{
DEVELOPMENTAL DISORDERS
}

PRADER-WILLI SYNDROME IN NEONATES

A retrospective study of 16 patients identifying physical features of neonates with Prader-Willi syndrome is reported from the Department of Pediatrics, Division of Genetics, William Beaumont Hospital, Royal Oak, MI and Section of Genetics, Department of Pediatrics, University of Arizona, Tucson. Medical records of 16 
patients observed in the clinic at the University of Connecticut Health Center, Farmington, were reviewed for descriptions made during the newborn period. At the time of the chart review the patients ranged in age from eight months to 33 years. All initial examinations were performed at 22 days of age or earlier. Of 13 patients with high resolution chramosome analysis 12 showed the typical deletion of the long arm of chromosame 15. Several of the characteristic features of Prader-Willi syndrame in early infancy were confirmed including hypotonia and genital hypoplasia. Features that have not previously been emphasized included an abnormal cry, disproportionately large head circumference and anterior fontanel, mild micrognathia, mild anomalies of gingivae or alveolar ridges, and changes in the appearance of the skin such as poor color, cyanosis, jaundice, ecchymoses, hirsutism, and foot edema. Hypoplasia of the scrotum was present with a normal appearing penis. Contrary to one previous report the hypotonia was associated with absence of deep tendon reflexes, and hyperreflexia was found in only one. (Aughton DJ, Cassidy SB. Physical features of Prader-Willi syndrome in neonates. AJDC Nov $1990 ; 144: 1251-1254)$.

OOMIENT. Prader-Willi syndrome is a sporadic multisystem disorder characterized after infancy by obesity, acromicria, short stature, hypogonadism, and abnormal cognitive and behavioral functioning. Infants exhibit hypotonia, genital hypoplasia, and feeding problems with failure to thrive. The additional neonatal characteristics described in this paper may aid in early diagnosis and counseling of parents.

NEURAL MATURATIONAL DELAY IN SIDS

The pathological evidence for developmental delay in SIDS is reviewed from the Division of Neuropathology, The Hospital for Sick Children, University of Toronto, Toronto, Canada. Evidence of hypoxic ischemic insult to the brain includes astrogliosis and subcortical leukamalacia. Astrogliosis is most apparent in the region of the tegmentum in SIDS victims, a finding interpreted as hypoperfusion during episodes of bradycardia associated with apnea. Subcortical or periventricular leukamalacia was found in $21.6 \%$ of infants who died of SIDS. Term and prematurely borm SIDS infants showed persistence of the reticular dendritic spines which suggests a delay of development to a mature, higher level of neuronal function. This delay may reflect a functional impaiment of the higher levels of respiratory control in SIDS infants. A number of reports suggest alterations in neurotransmitter levels of catecholamines, beta endorphin, metenkephalin, and substance $P$ in SIDS. Examination of 36 infants who had died of SIDS showed that the mean number of small myelinated vagal fibers was significantly decreased in the SIDS infants compared with controls, suggesting an abnormal or delayed development of the vagus nerve. This finding was similar to that reported in a two year old infant dying of persistent infantile sleep apnea (Ondine's curse). Elevated dopamine in the carotid bodies of SIDS victims suggests a role in pathogenesis: dopamine inhibits respiration by acting directly on 